Journal for ImmunoTherapy of Cancer

\section{Delivery of CD47 blocker SIRPa-Fc by CAR-T cells enhances antitumor efficacy}

To cite: Chen H, Yang Y, Deng Y, et al. Delivery of CD47 blocker SIRP $\alpha$-Fc by CAR-T cells enhances antitumor efficacy. Journal for ImmunoTherapy of Cancer 2022;10:e003737. doi:10.1136/jitc-2021-003737

- Additional supplemental material is published online only. To view, please visit the journal online (http://dx.doi.org/10. 1136/jitc-2021-003737).

Accepted 08 January 2022

Check for updates

(C) Author(s) (or their employer(s)) 2022. Re-use permitted under CC BY-NC. No commercial re-use. See rights and permissions. Published by BMJ.

${ }^{1}$ BioResource Research Center, Key Laboratory for Major Obstetric Diseases of Guangdong Province, The Third Affiliated Hospital of Guangzhou Medical University, Guangzhou, Guangdong, China

${ }^{2}$ Institute of Human Virology, Sun Yat-sen University Zhongshan

School of Medicine, Guangzhou, Guangdong, China

${ }^{3}$ Key Laboratory of Tropical

Disease Control, Sun Yat-

Sen University, Guangzhou, Guangdong, China

${ }^{4}$ Faculty of Science, Monash University, Clayton, Victoria, Australia

${ }^{5}$ ICU, Sun Yat-sen University Cancer Center, Guangzhou, Guangdong, China

${ }^{6}$ Laboratory Animal Center, South China Agricultural University, Guangzhou, Guangdong, China

Correspondence to Dr Zhaofeng Huang; hzhaof@mail.sysu.edu.cn

\section{ABSTRACT}

Background Chimeric antigen receptor (CAR) T cell therapy has been successfully applied in treating lymphoma malignancies, but not in solid tumors. CD47 is highly expressed on tumor cells and its overexpression is believed to inhibit phagocytosis by macrophages and dendritic cells. Given the antitumor activity against preclinical model of CD47-blocking to induce the innate and adaptive immune system in the tumor microenvironment, here we developed a CAR-T cell secreting CD47 blocker signal regulatory protein $\alpha$ $(\mathrm{SIRP} \alpha)$-Fc fusion protein (Sirf CAR-T) to boost CAR-T cell therapeutic effect in solid tumor therapy.

Methods Murine T cells were transduced to express a conventional anti-Trop2 CAR and Sirf CAR. The expression of SIRP $\alpha$-Fc fusion protein in the supernatant of CAR-T cells and its effect on macrophage phagocytosis were tested in vitro. In vivo antitumor efficacy of CAR-T cells was evaluated in immunocompetent mice and analysis of the tumor microenvironment in the tumor-bearing mice was performed.

Results We found that Sirf CAR-T cells dramatically decreased tumor burden and significantly prolonged survival in several syngeneic immunocompetent tumor models. Furthermore, we found that Sirf CAR-T cells induced more central memory $T$ cells $\left(T_{C M}\right)$ and improved the persistence of CAR-T cells in tumor tissue, as well as decreased PD-1 expression on the CAR-T cell surface. In addition, we demonstrated that Sirf CAR-T cells could modulate the tumor microenvironment by decreasing myeloid-derived stem cells as well as increasing CD $11 \mathrm{c}^{+}$ dendritic cells and M1-type macrophages in tumor tissue. Conclusions In summary, our findings indicate that CD47 blocker SIRP $\alpha$-Fc enhances the antitumor efficacy of CAR-T cells and propose to block CD47/SIRP $\alpha$ signaling effect on CAR-T cells function, which could provide a new strategy for successful cancer immunotherapy by rationalizing combination of CD47 blocker and CAR-T cell therapy.

\section{BACKGROUND}

Chimeric antigen receptor (CAR) $\mathrm{T}$ cell therapy is an attractive adoptive cellular therapy tactic to the field of individualized cancer immunotherapy. ${ }^{1}$ CAR-T cells could recognize and bind to the tumor antigen in a major histocompatibility complex (MHC) independent manner to eliminate tumor cells. CAR is a synthetic receptor consists of an extracellular antigen-binding domain, which is often a single-chain fragment variant $(\mathrm{scFv})$ derived from a $\mathrm{mAb}$, linked to various costimulatory domains and $\mathrm{CD} 3 \zeta$ intracellular signaling domain to induce T cell activation. ${ }^{2}$ Adoptive transfer of CAR-T cells has led to a revolution in the treatment of $\mathrm{B}$ cell hematological malignancies and yielded remarkable clinical responses in other patients with refractory hematological malignancies. ${ }^{3}$ CAR-T cell therapy has emerged as a promising approach in cancer treatment. There are currently five FDA-approved CAR-T cell therapies, targeting the CD19 or BCMA molecule, in the treatment of relapsed refractory B-cell acute lymphoblastic leukemia, large B-cell non-Hodgkin lymphoma, relapsed refractory mantle cell lymphoma, and relapsed refractory multiple myeloma. ${ }^{4-7}$

Nowadays, several clinical trials of CAR-T cell therapy have shown tremendous success in treating hematologic malignancies. However, due to the tumor heterogeneity and intricacies of the tumor microenvironment (TME), its efficacy in treating solid tumors has not yet been supported. ${ }^{8}$ TME often contains many immunosuppressive cells, such as regulatory $\mathrm{T}$ cell (Treg), myeloid-derived stem cell (MDSC), and tumor-associated macrophages (TAMs), as well as the immune inhibitory molecules in tumor tissues. ${ }^{9}$ All of these significantly impaired the antitumor effects of CAR-T cells treating against solid tumors. ${ }^{10-12}$ Biological obstacles also limited the success of using CAR-T cells for the treatment of solid tumors, such as poor $\mathrm{T}$ cell persistence, $\mathrm{T}$ cell exhaustion, and insufficient homing of infused $\mathrm{T}$ cells to tumor sites. $^{13}$ Currently, some efforts have been made to improve the CAR-T cell antitumor activity to address these obstacles during solid tumor treatment, including coexpression of IL-12, IL-18, IL-7 with CCL19, and CCR2b in the CAR-T cells. ${ }^{14-17}$ These explorations tried to combine the antitumor effect of CAR-T cells with increasing immune-checkpoint 
blockade as well as the suppression of other inhibitors in the TME to enhance the antitumor activity of CAR-T cells, and some of them shed the promise that will be verified in clinical practice. ${ }^{18}$ To against heterogeneous and immunosuppression of solid tumors, more strategies are urgently required to improve the antitumor effect of CAR-T cells for cancer therapy.

CD47 is a transmembrane glycoprotein widely expressed on normal cells and highly expressed on the surface of tumor cells. ${ }^{19} \mathrm{CD} 47$ binds to the signal regulatory protein $\alpha(\operatorname{SIRP} \alpha)$ on the surface of macrophages to inhibit their phagocytic ability, thereby helping tumor cells to evade the recognition and surveillance of the immune system. ${ }^{20}$ High expression of $\mathrm{CD} 47$ is associated with poor clinical prognosis. ${ }^{21}$ Blocking the CD47-SIRP $\alpha$ signal axis can enhance the in vitro tumor phagocytic capacity of macrophages and lead to a depletion of M2 macrophages. ${ }^{22}$ CD47 blockade not only affects macrophages function, but also induces dendritic cells (DC) maturation. Their antigen presenting capacity benefits in enhancing the function of adaptive immunity synergized with $\mathrm{CD}^{+} \mathrm{T}$ cells. ${ }^{23}$ Therefore, therapeutic approaches to repolarize or enhance the phagocytic activity of TAMs are important in clinical development. CD47 blocking antibodies have been used in both hematoma and solid tumors, but the side effects of the antibody's blood toxicity and other side effects limit its further efficacy. ${ }^{24}$ Furthermore, clinical trials have also shown that except for a few hematomas, CD47-targeted monotherapy has limited efficacy on most solid tumors. ${ }^{25}$ It is very necessary to further explore the combination of CD47 blockade with other immunotherapies for solid tumor therapy.

We previously described that Trop2 can be used as a target for CAR-T cells and demonstrated the antitumor effect of murine T2-CAR T cells. ${ }^{26}$ Here, we investigated whether the combination of CAR-T cells with CD47 blockade could improve the antitumor activity. We developed Sirf CAR-T cells secreting CD47 blocker, SIRPo-Fc protein, to enhance the phagocytosis of macrophages and therapeutic effects of CAR-T cells in solid tumors. This study will reveal the potential for a combination of SIRP $\alpha-F c$ protein with CAR-T cells in solid tumor therapy.

\section{MATERIALS AND METHODS \\ Retroviral vectors design and construction}

The human Trop2-specific murine CAR (T2-m28z) was generated by linking antihuman Trop2 scFv to the mouse CD8 $\alpha$ hinge domain, mouse CD28 transmembrane and the cytoplasmic domain, and the intracellular signaling domain of mouse CD3 $\zeta$. CAR genes were then cloned into the retroviral vector MIGR1 which was used as previously described. ${ }^{26}$ Anti CD47 fusion protein (SIRP $\left.\alpha-F c\right)$ consists of the N-terminal V domain of mouse SIRP $\alpha$ linked to the mouse IgG2a Fc which was analogous to the human IgG1 Fc region for full effector function. The SIRP $\alpha$-Fc protein was tagged at the C-terminus with a HIS tag.To construct a vector encoding SIRP $\alpha$-Fc in murine CAR (Sirf), the
SIRP $\alpha-F c$ gene was inserted into the BamHI and ClaI restriction enzyme sites of the MIGR1 retroviral vector, and 2A self-cleavable peptide from porcine teschovirus-1 polyprotein was inserted between T2-m28z and SIRP $\alpha-F c$.

\section{CAR-T cell generation}

Retrovirus production and mouse CAR T cells transduction was conducted as described previously. Briefly, 293T cells were transfected with the CAR-expressing retrovirus plasmid and pCL-Eco packaging plasmid by using calcium phosphate method. The culture supernatants were harvested and frozen for gene transduction after 48 hours. Mouse T cells isolated from wild-type C57BL/ 6 or $\mathrm{BALB} / \mathrm{c}$ female mouse splenocytes were purified with a mouse $\mathrm{CD}^{+} \mathrm{T}$ cell negative enrichment kit (BD, USA). $\mathrm{T}$ cells were activated in $0.2 \mathrm{mg} / \mathrm{mL}$ rabbit anti-hamster (A18897; Invitrogen) precoated 24-well plate, cultured in RPMI 1640 (Invitrogen) containing $2 \mathrm{mM}$ l-glutamine, $50 \mathrm{mM}$ 2-ME, $100 \mathrm{U} / \mathrm{mL}$ penicillin, $100 \mathrm{mg} / \mathrm{mL}$ streptomycin and $10 \%$ fetal bovine serum (FBS) supplemented with $0.25 \mu \mathrm{g} / \mathrm{mL}$ hamster anti-CD3 (145-2 C11; eBioscience; 16-0031-85), $1 \mu \mathrm{g} / \mathrm{mL}$ hamster anti-CD28 (37.51; eBioscience; 16-0281-85) and $10 \mathrm{ng} / \mathrm{mL}$ murine IL-2 (R\&D) for 24 hours. The activated T cells were then infected with retroviral supernatants in the presence of RetroNectin (Takara Bio) and transduction efficacy was determined in $\mathrm{GFP}^{+}$with flow cytometry analysis.

\section{Cell lines}

A total of 293T cells were obtained from ATCC (Manassas, Virginia, USA) and maintained in conditioned highglucose DMEM (Gibco, Invitrogen, Carlsbad, California, USA) supplemented with 10\% Fetus Bovine Serum(FBS) and $100 \mathrm{U} / \mathrm{mL}$ penicillin/streptomycin (Invitrogen). MC38, a colorectal cancer cell derived from C57BL/6, and $4 \mathrm{~T} 1$, breast cancer cell derived from BALB/c, were transduced with recombinant retroviruses carrying Trop2IRES-GFP moiety to establish MC38-Trop2 ${ }^{+}$and 4T1Trop2 ${ }^{+}$cells, followed by GFP ${ }^{\text {high }}$ sorting (BD FACSAria II). MC38-Trop2 ${ }^{+}$and 4T1-Trop2 ${ }^{+}$cells were cultured in RPMI 1640 (Invitrogen) containing 10\% FBS and 100 $\mathrm{U} / \mathrm{mL}$ penicillin/streptomycin. All cell lines were maintained in a humidified atmosphere containing $37^{\circ} \mathrm{C}$ and $5 \% \mathrm{CO}_{2}$.

\section{Flow cytometry}

The following Abs were used in flow cytometry analysis: PE conjugated anti-mouse TCR (clone H57-597, Invitrogen), APC conjugated anti-mouse CD8 (clone 53-6.7, Invitrogen), Pacific Blue conjugated anti-mouse CD45 (clone 30-F11, Invitrogen), PerCP-Cyanine5.5 conjugated anti-mouse CD4 (clone RM4-5, Invitrogen), SB600 conjugated anti-mouse CD8 (clone 53-6.7, Invitrogen), PE-Cyanine7 conjugated anti-mouse CD3 (clone 145-2 C11, Invitrogen), APC conjugated anti-mouse PD-1 (clone J43, Invitrogen), Pacific Blue conjugated anti-mouse CD4 (clone RM4-5, Invitrogen), PE-Cyanine7 conjugated anti-mouse CD47 (clone miap301, Invitrogen), 
PerCP-Cyanine5.5 conjugated anti-mouse CD11b (clone $\mathrm{M} 1 / 70$, Invitrogen), PE conjugated anti-mouse F4/80 (clone BM8, Invitrogen), PE conjugated anti-mouse IFN- $\gamma$ (clone XMG1.2, Invitrogen), PerCP-Cyanine5.5 conjugated anti-mouse CD11c (clone N418, TONBO Biosciences), PerCP-Cyanine5.5 conjugated anti-mouse CD44 (clone IM7, Invitrogen), APC-eFlour780 conjugated anti-mouse CD62L (clone MEL-14, Invitrogen), PE-Cyanine7 conjugated anti-mouse CD206 (clone MR6F3, Invitrogen), APC conjugated anti-mouse MHCII (clone AF6-88.5.5.3, Invitrogen), APC conjugated anti-mouse Ly6G (clone 1A8-Ly6g, Invitrogen), PE conjugated antimouse Ly6C (clone HK1.4, Invitrogen) FACS analysis was performed with flow cytometry (LSR Fortessa, BD) and FlowJo software (FlowJo_V10, BD). An Annexin V-PE Apoptosis Detection Kit (BD Pharmingen) was used to evaluate apoptosis levels according to the manufacture's guidelines.

\section{In vitro cytotoxicity and activation assays of CAR-T cells}

The cytotoxic activity of the CAR-T cells was evaluated using the CytoTox 96 Non-Radioactive Cytotoxicity Assay (G1781, Promega) following manufacture guidelines. The lactate dehydrogenase (LDH) release was evaluated after 8 hours in the supernatant with effector-to-target ratios of 1:1, 2:1, and 5:1.

IFN- $\gamma$ expression was detected by intracellular cytokine staining. CAR-T cells were cocultured with tumor cells at a 2:1 ratio, with $1 \times 10^{6} \mathrm{~T}$ cells seeded in 12-well flatbottom tissue culture plates for 8 hours. Then brefeldin A inhibitor was added and cells were harvested 6 hours later and stained with anti-mouse CD3 antibody. The cells were then fixed, permeabilized, and stained with anti-mouse IFN- $\gamma$ according to the manufacture's guidelines (88-8824-00, eBioscience Intracellular Fixation \& Permeabilization Buffer Set).

For the tests of $\mathrm{T}$ cell activation and apoptosis in vitro, $5 \times 10^{5}$ CAR-T cells were stimulated with $5 \times 10^{5}$ MC38Trop2 cells in 12-well tissue culture plates. After 48 hours, CAR-T cells were harvested to assess the level of apoptosis, expression of memory-related proteins, and exhaustion markers.

\section{Animal models}

All studies complied with the ethical regulations established by the Institutional Animal Care and Use Committee of South China Agricultural University. To assess the antitumor effects of CAR-T cells in the established MC38-Trop2 ${ }^{+}$mice tumor models, $6-8$ weeks old female C57BL/6 mice were inoculated subcutaneously (s.c.) with $5 \times 10^{5}$ tumor cells in the right flank $(0 \mathrm{~d})$. At 7 d, $1 \times 10^{6}$ CAR-T cells were injected intravenously. In some experiments, C57BL/6 mice were inoculated s.c. with $5 \times 10^{5}$ MC38-Trop2 $2^{+}$tumor cells on $0 \mathrm{~d}$, and then cyclophosphamide (CY: $80 \mathrm{mg} / \mathrm{kg}$, Sigma) was administered intraperitoneally (i.p.) $14 \mathrm{~d}$ after tumor inoculation. On day $17,1 \times 10^{6}$ cells CAR-T cells were injected intravenously. In the $4 \mathrm{~T} 1-\mathrm{Trop} 2^{+}$model, BALB/c mice were inoculated (s.c.) with $5 \times 10^{5}$ tumor cells in the right flank $(0 \mathrm{~d})$, and then injected intravenously with $1 \times 10^{6} \mathrm{CAR}-\mathrm{T}$ cells at $2 \mathrm{~d}$ and $7 \mathrm{~d}$. In some experiments, mice were inoculated (s.c.) with $5 \times 10^{5}$ MC38-Trop2 ${ }^{+}$cells $(0 \mathrm{~d})$, and then injected intravenously with $1 \times 10^{6}$ CAR-T cells at 14 d. Tumor size was measured by digital calipers. On day 21, spleen cells and tumor-infiltrating lymphocytes were harvested to assess the ratio and kinetics of the CAR-T cells.

\section{Preparation of SIRP $\alpha-F c$ fusion protein}

The coding sequences of SIRP $\alpha$-Fc with HIS-tag were cloned into the mammalian expression vector pcDNA3.1 and the sequences were verified by Sanger sequencing. For transient transfection-based protein production, expressing vectors were transfected into $293 \mathrm{~T}$ cells using calcium phosphate method. Cell culture media containing SIRP $\alpha$-Fc were harvested on day 5 by centrifugation at $4000 \mathrm{rpm}$ for $5 \mathrm{~min}$, followed by Protein A affinity chromatography purification.

\section{Generation of bone marrow-derived macrophages}

Murine whole bone marrow cells were isolated from 8 to 10 weeks old C57BL/6 mice. Bone marrow-derived macrophages were generated by incubating bone marrow cells in Dulbecco's Modified Eagle Medium (DMEM) supplemented with $10 \%$ fetal bovine serum (FBS) and recombinant Murine granulocyte-macrophage colony stimulating factor (GM-CSF) $(20 \mathrm{ng} / \mathrm{mL}$, Peprotech) for 7 days. Meanwhile, the medium was replaced with fresh cytokine-containing on days 2 and 4 . Nonadherent cells were washed and adherent cells were harvested on day 7 .

\section{In vitro phagocytosis assay}

For the in vitro phagocytosis assay, macrophages cocultured with MC38 tumor cells which were labeled with carboxyfluorescein diacetate succinimidyl ester at a 1:5 ratio in serum-free RPMI 1640 medium at $37^{\circ} \mathrm{C}$ for 4 hours. To further demonstrate whether mouse SIRP $\alpha$-Fc protein in $\mathrm{T}$ cells supernatant directly contributes to the tumor phagocytosis of macrophages, different $\mathrm{T}$ cell supernatants or $\mathrm{T}$ cell supernatants co-cultured with MC38-Trop2 $2^{+}$tumor cells were added to the macrophage and tumor cell co-cultivation system. Then macrophages were washed with PBS and subsequently stained with antimouse F4/80, anti-mouse CD11b, and 7-AAD for flow cytometry analysis. The percentage of phagocytosis was assessed as the percentage of $\mathrm{CFSE}^{+}$cells in $\mathrm{F} 4 / 80^{+}$and CD11 $b^{+}$macrophages.

\section{Statistical analysis}

Data were analyzed using Prism (V.7.0, GraphPad) software and the results were presented as mean \pm SEM where indicated. Statistical significance was determined by using a one-way analysis of variance (ANOVA) with Dunnett's test or a two-way ANOVA analysis. Survival curves were calculated by implementing a Log-rank (Mantel-Cox) test. $* \mathrm{p}<0.05, * * \mathrm{p}<0.01, * * * \mathrm{p}<0.001, * * * * \mathrm{p}<0.0001$ were considered significant. 
A
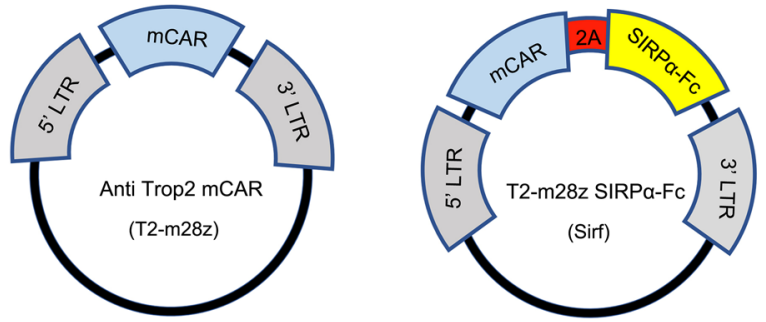

B
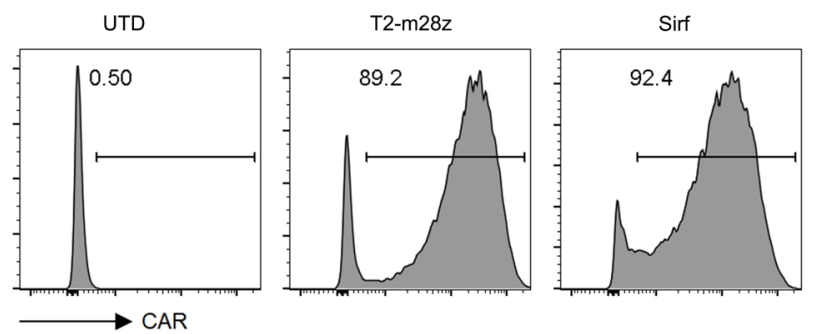

C
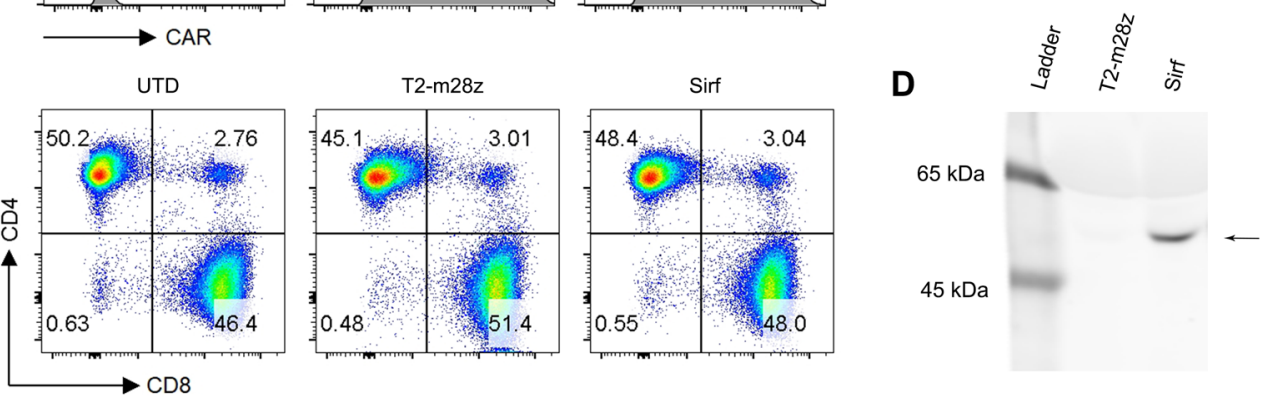

E
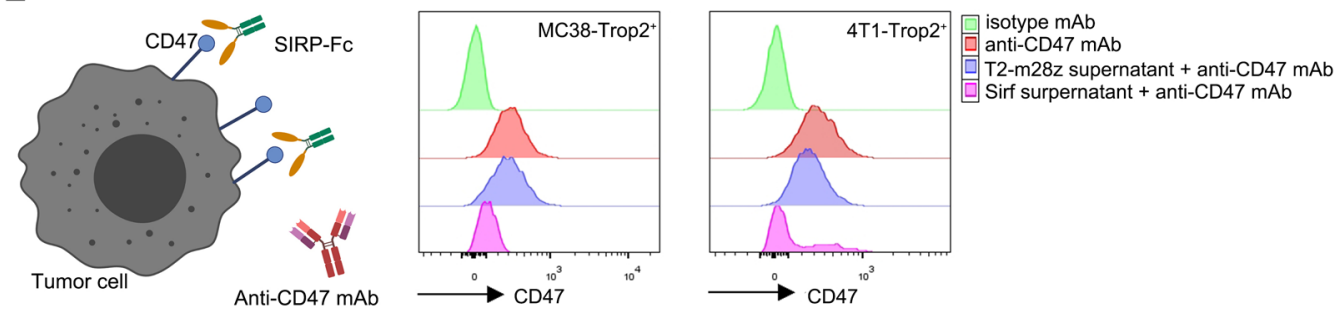

Figure 1 Generation and functional characterization of mouse CAR-T cells coexpression of SIRP $\alpha$-Fc. (A) The schemas diagram of second-generation mouse CAR (T2-m28z) and Sirf CAR (T2-m28z SIRP $\alpha-F c)$ structures. (B) CAR expressions were analyzed by using flow cytometry, and untransduced (UTD) T cell was used as the negative control. (C) The ratio of CD4 ${ }^{+}$and $\mathrm{CD}^{+}$in the T cell subsets was detected. (D) Western blotting analysis of the SIRP $\alpha-F c$ fusion protein secretion in supernatant of T2-m28z and Sirf CAR-T cells. (E) Flow cytometry assay detected the expression of CD47 on MC38-Trop ${ }^{+}$and 4T1-Trop2 ${ }^{+}$ tumor cells. Tumor cells were stained with a fluorescent isotype antibody (green) or a fluorescent anti-CD47 mAb (red). After treated with supernatant of T2-m28z CAR-T cells (purple) or Sirf CAR-T cells (pink), tumor cells were then stained with fluorescent anti-CD47 mAb. CAR, chimeric antigen receptor; SIRP $\alpha$, signal regulatory protein $\alpha$.

\section{RESULTS \\ Modification of mouse CAR-T cells to secrete CD47 blocker SIRP $\alpha-F c$}

CAR-T cell (T2-m28z) targeting human Trop2 that have been demonstrated antitumor function in previous study. ${ }^{26}$ To explore the effectiveness of CD47 blocker SIRP $\alpha$ enhancing CAR-T cell treatment in cancer therapy. SIRPo-Fc-secreting mouse CAR (Sirf) were generated by using the T2-m28z CAR constructs connected with SIRP $\alpha-F c$ by self-cleavable 2A peptide linker (figure 1A). The retroviral transduction efficiency of T2-m28z and Sirf CAR-T cells was around 90\% (figure 1B). The ratio of $\mathrm{CD}^{+}$to $\mathrm{CD}^{+}$in the prepared CAR-T cells was similar (figure 1C), which indicated that the SIRP $\alpha-F c-s e c r e t i n g$ fusion protein did not affect the ratio of $\mathrm{CD} 4^{+} \mathrm{T}$ cells and $\mathrm{CD}^{+} \mathrm{T}$ cells. The secretion of SIRP $\alpha-\mathrm{Fc}$ in CAR-T cells was further confirmed by performing anti-His immunoblotting on the culture supernatant of CAR-T cells, and the target protein was present in the supernatant of Sirf CAR-T cells but not in T2-m28z (figure 1D). To evaluate whether the SIRP $\alpha-F c$ in the supernatant of Sirf CAR-T cells could bind to the CD47 on the surface of tumor cells, a flow cytometry-based antibody competitive assay was used. It was found that CD47 was highly expressed on MC38-Trop2 and 4T1-Trop2 tumor cells at baseline. The fluorescence intensity of CD47 staining was significantly reduced after co-incubation with Sirf CAR-T cell supernatant but not with T2-m28z CAR-T cells (figure 1E). These results demonstrated that Sirf CAR-T cells secreted SIRP $\alpha$-Fc protein which could bind to the CD47 molecule on tumor cells. 
A

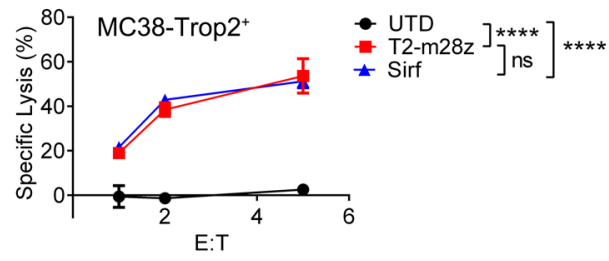

B

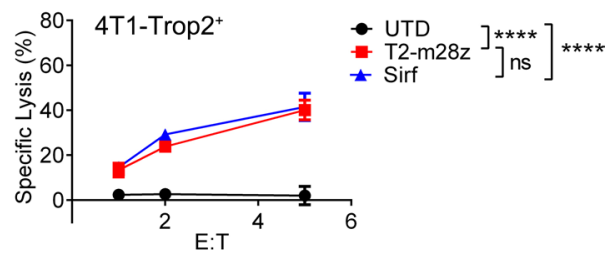

C
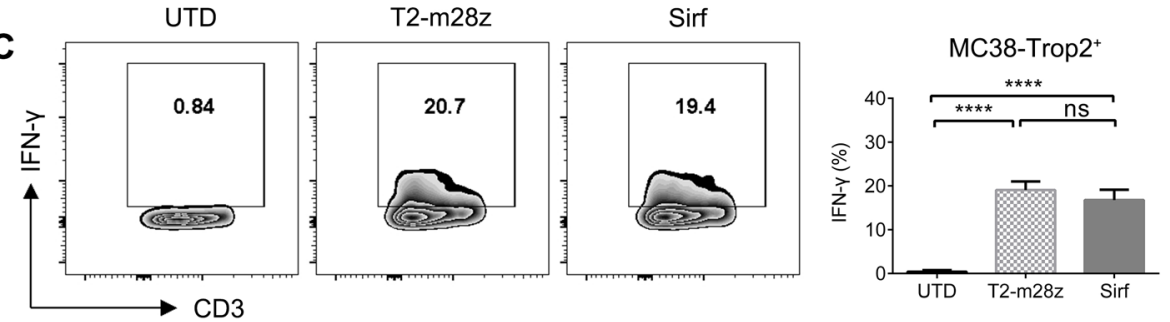

D
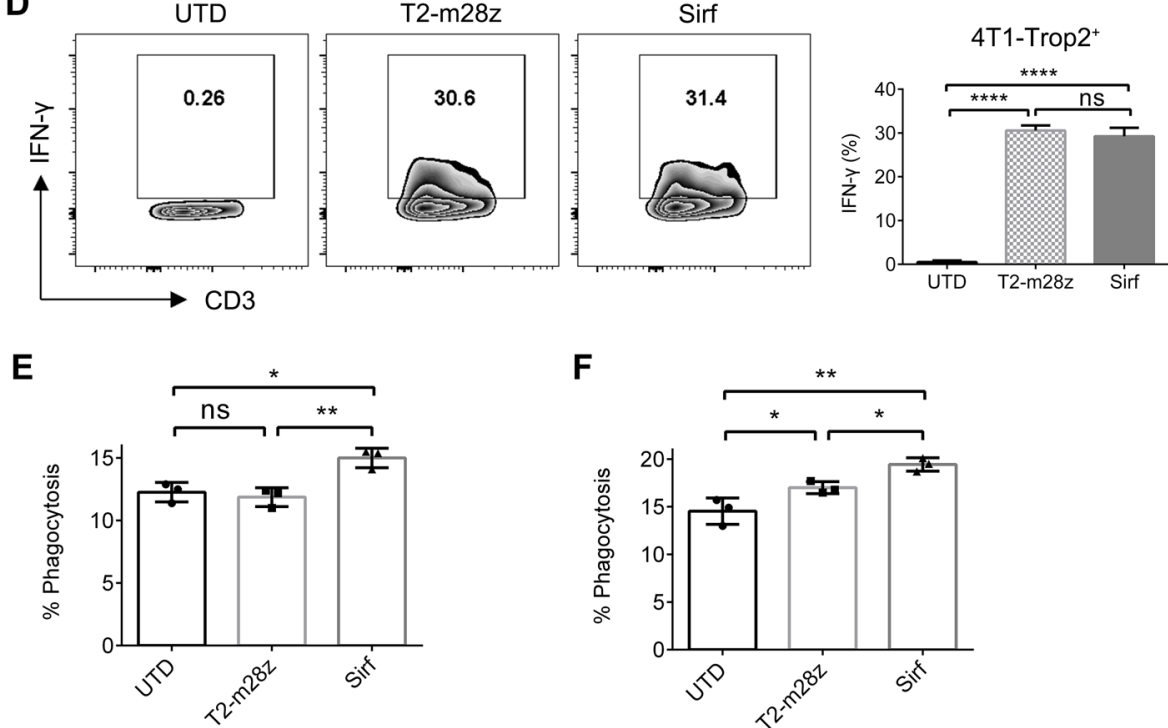

Figure 2 In vitro antitumor function of CAR-T cells. (A, B) The cytotoxic effect of CAR-T cells with different trop2-positive murine tumor cells. The expression of IFN- $\gamma$ in $\mathrm{CD}^{+}{ }^{+}$cells when cocultured with MC38-Trop2 ${ }^{+}$cells $(C)$, and 4T1-Trop2 ${ }^{+}$cells (D) were conducted by flow cytometry. For the in vitro phagocytosis assay, BMDMs were cocultured with CFSE-labeled MC38Trop $^{+}$cells at a 1:4 ratio in the supernatant of different T cells $(E)$ or the medium of cocultured T cells with tumor cells $(F)$. The cells were harvested after 4 hours coculture, and primary macrophages were identified by flow cytometry using anti-F4/80. Phagocytosis rate was determined as the percentage of $\mathrm{CFSE}^{+}$macrophages. (mean $\pm \mathrm{SEM}, n=3$ ). Representative data from at least three independent experiments are shown. ${ }^{*} p<0.05,{ }^{\star \star} p<0.01,{ }^{\star \star \star \star} p<0.0001$. BMDMs, bone marrow-derived macrophages; CAR, chimeric antigen receptor; CFSE, carboxyfluorescein diacetate succinimidyl ester; ns, not significant; UTD, untransduced.

\section{Effect of SIRP $\alpha-F c$ secretion on tumor lysis of CAR-T cells and macrophage phagocytosis in vitro}

To determine whether SIRP $\alpha-F c$ influences the killing ability of CAR-T cells, we next performed LDH release assay and revealed that both Sirf and T2-m28z CAR-T cells showed similar tumor-lytic ability against MC38Trop2 $^{+}$cells and 4 T1-Trop2 ${ }^{+}$cells (figure 2A,B), as well as the expression of IFN- $\gamma$ (figure 2C,D). These demonstrated that secretion of SIRP $\alpha$-Fc would have no adverse effect on the antitumor effects of CAR-T cells in vitro. To determine the effect of secreted SIRP $\alpha$-Fc by CAR-T cells on macrophages' phagocytosis, we tested the phagocytosis ability against tumor cells after supplement with different CAR-T cell culture supernatant. Results showed that Sirf CAR-T cell supernatant could enhance phagocytosis compared with conventional ones (figure $2 \mathrm{E}$ ). In addition, CAR-T cells could induce IFN- $\gamma$ expression after stimulated by tumor antigen. To determine whether IFN- $\gamma$ expressed by CAR-T cells could enhance macrophagemediated phagocytosis of mouse tumor cells, we analyzed the macrophage phagocytosis after supplementing with supernatant from different CAR-T cells coculturing with MC38-Trop2 ${ }^{+}$cells. A marked prophagocytic effect was observed on Sirf CAR-T cells supernatant than that of the UTD or conventional CAR-T cells (figure 2F). Collectively, these in vitro data indicated that secreted SIRP $\alpha-F c$ protein enhances the macrophage phagocytosis but not impair CAR-T cell activity. 
A

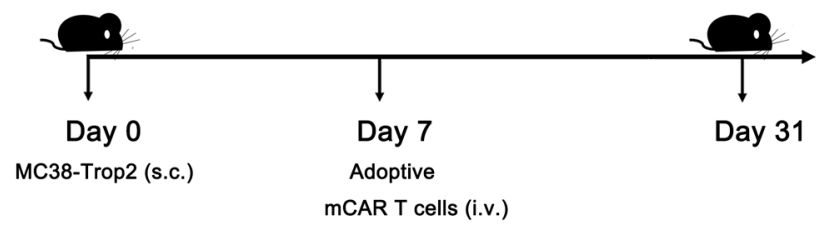

B

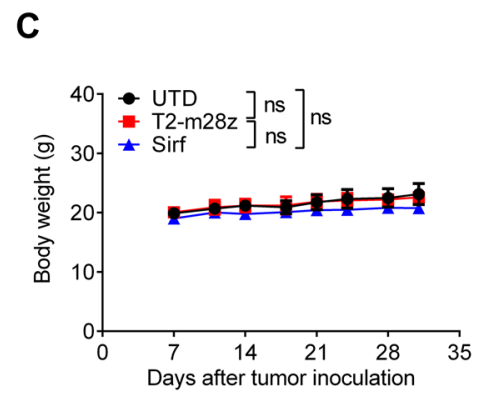

D

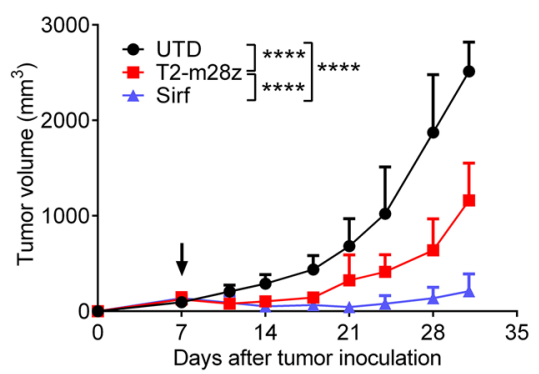

E
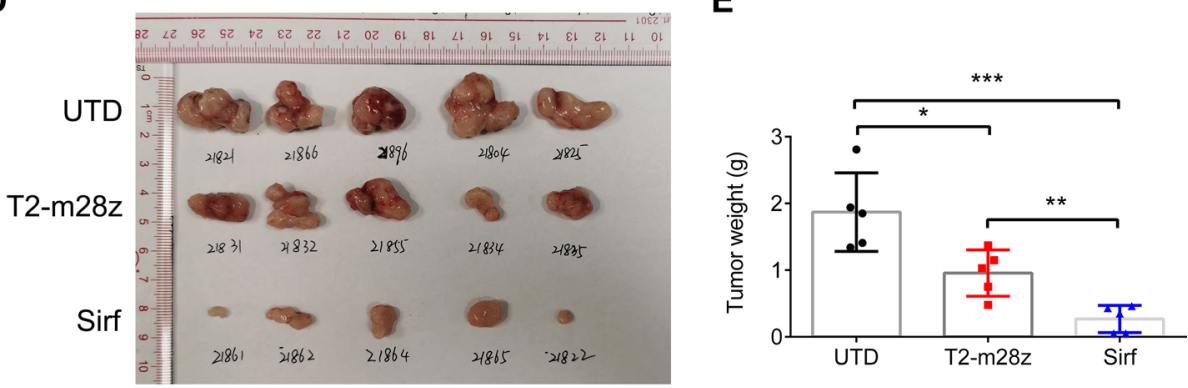

Figure 3 Antitumor effects of Sirf CAR-T cells on mouse colon cancer in vivo. (A) Schematic representation of the animal experiment. (B) Tumor growth curve of mice. (C) The changes in the body weight of tumor-bearing mice. (D) The pictures of resected tumor mass on day 31 at the endpoint. (E) The weights of individual tumors are presented. N=5 mice/group; All data are presented as mean \pm SEM from experiments. ${ }^{\star} p<0.05,{ }^{\star \star} p<0.01,{ }^{\star \star \star} p<0.001,{ }^{\star \star \star \star} p<0.0001$. CAR, chimeric antigen receptor; ns, not significant; s.c, subcutaneously; UTD, untransduced.

\section{Enhanced antitumor activity of Sirf CAR-T cells in vivo}

We next investigated antitumor activity of Sirf CAR-T cells in the immunocompetent mouse model. C57BL/6 mice were inoculated with MC38-Trop2 ${ }^{+}$(s.c.) and then treated with Sirf CAR-T cells, T2-m28z CAR-T cells, or activated $\mathrm{T}$ cells without gene transfection (figure 3A). As compared with control, both T2-m28z CAR-T and Sirf CAR-T cells provided a moderate reduction in tumor growth (figure $3 \mathrm{~B}$ ). Additive control of tumor growth was observed in mice treated with Sirf CAR-T cells compared with T2-m28z CAR-T cells. The treatment was not associated with potential toxicity as demonstrated by body weight changes (figure 3C). To further examine the antitumor efficacy of CAR-T cells, tumors were excised on day 31 (figure 3D) and the tumor mass dramatically reduced in the Sirf CAR-T cell treatment group (figure 3E). SIRP $\alpha-F c$ recombinant fusion protein were produced and purified as the $\mathrm{CD} 47$ blockade (online supplemental figure S1). To identify its therapeutic effects for solid tumor, the administration of SIRP $\alpha-\mathrm{Fc}$ recombinant fusion protein was performed in an in vivo experiment, in which it displayed no inhibition on tumor growth (online supplemental figure S2). The combination of SIRP $\alpha-F c$ fusion protein with T2-m28z CAR-T cells could not enhance the therapy effect of conventional CAR-T cells. Compared with other treatments, injection of Sirf CAR-T cells led to the best tumor growth inhibition effect (figure 3, online supplemental figure S2). These results indicate an enhanced antitumor effect of Sirf CAR-T cells in vivo.

In many clinical practices, low-dose fludarabine or CY pretreatment is usually required for CAR-T cells treatments. To determine the combination therapeutic effects of CAR-T cells with CY, mice were inoculated with MC38Trop2 ${ }^{+}$(s.c.), employed with CY (i.p.) pretreatment, and then treated by intravenously injection with CAR-T cells (figure 4A). Following this therapeutic procedure, the administration of Sirf CAR-T cells resulted in remarkable prolongation of mouse survival (figure $4 \mathrm{~B}$ ) and reduction of tumor burden (figure 4C). Moreover, the enhanced therapeutic effects of Sirf CAR-T cells were further confirmed in another tumor-bearing model (figure 4D). We observed a similar result on 4T1-Trop2 ${ }^{+}$ tumor growth. Treatment with Sirf CAR-T cells induced a significant reduction in tumor volume, while T2-m28z CAR-T cells showed only a limited effect (figure 4E). Taken together, these results indicated that Sirf CAR-T cells could improve therapeutic effects against solid tumors. 
A

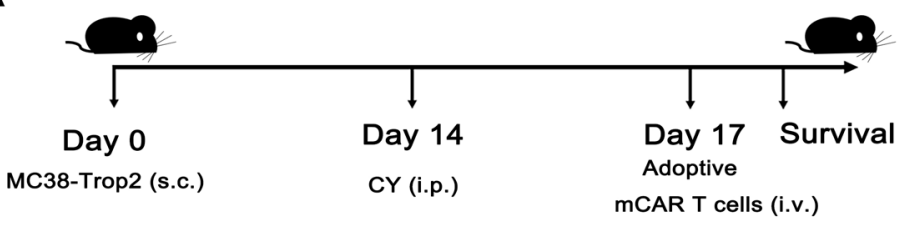

B

C
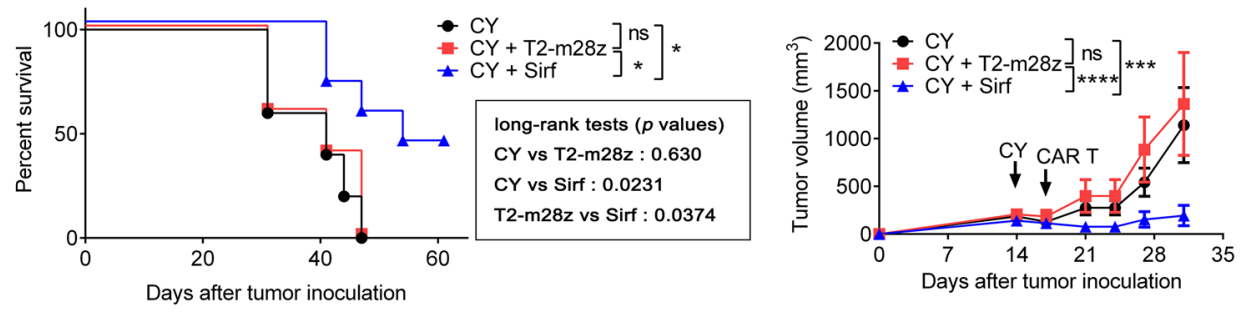

D

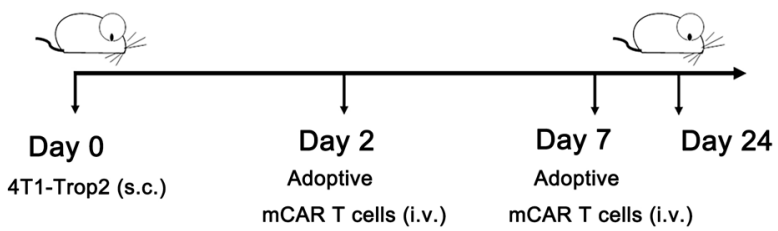

E

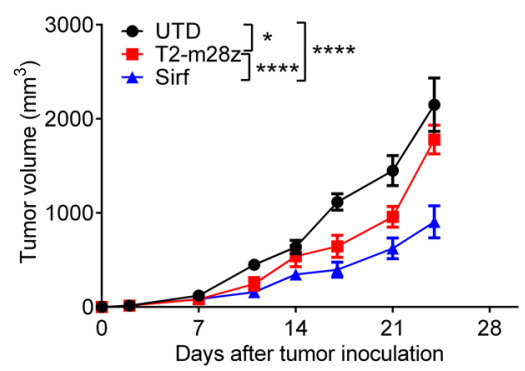

Figure 4 Improved therapeutic effects of Sirf CAR-T cells against pre-established solid tumor model. (A) C57BL/6 mice were inoculated subcutaneously with MC38-Trop ${ }^{+}$cells and treated with $\mathrm{CY}$ before CAR-T cells treatment. (B) Survival of the mice and the volume of the tumors $(\mathrm{C})$ were shown ( $\mathrm{N}=5, \mathrm{CY}$ group; $\mathrm{N}=5, \mathrm{~T} 2-\mathrm{m} 28 \mathrm{z}$ group; $\mathrm{N}=7$, Sirf group). All data are presented as mean \pm SEM from experiments. P values were calculated by Log-rank (Mantel-Cox) test. (D) Schematic representation of BALB/c mice were inoculated subcutaneously (s.c.) with $4 \mathrm{~T} 1-T r o p 2^{+}$cells. (E) Tumor growth curve of mice. $\mathrm{N}=5$ mice/group. All data are presented as mean \pm SEM from experiments. ${ }^{*} p<0.05,{ }^{* \star \star *} p<0.0001$. CAR, chimeric antigen receptor; $C Y$, cyclophosphamide;

UTD, untransduced.

\section{SIRP $\alpha$-Fc is beneficial for CAR-T cells to reduce immunosuppression}

We next performed flow cytometry analyses to investigate whether secreted SIRP $\alpha-F c$ can also improve the functions of the immune system and modulate the tumor immune microenvironment in tumor-bearing mice. We observed that the proportion of CAR-T cells in spleen $\mathrm{CD}^{+} \mathrm{T}$ cells in Sirf treated mice were higher than that in the T2-m28z group, and CD8 ${ }^{+}$CAR-T cells were the predominant ones (figure 5A-C). Moreover, Sirf CAR-T cells enhanced the proportion of $\mathrm{CD} 44^{+} \mathrm{CD} 62 \mathrm{~L}^{+}$central memory $\mathrm{T}$ cell $\left(\mathrm{T}_{\mathrm{CM}}\right)$ in mice splenocytes (figure $5 \mathrm{D}$ ). As expected, we also found that the proportion of CAR-T cells were significantly higher in tumor tissues treated with Sirf CAR-T cells than in the control group (figure 5E,F). In addition, we observed increased proportion of $\mathrm{CD} 11 \mathrm{c}^{+}$DC (figure $5 \mathrm{G}$ ) and a decrease occurred in the proportion of myeloid-derived suppressor cells (MDSC) in the tumor tissues treated with Sirf CAR-T cells (figure 5H). Since CD47 blockage enhanced phagocytic capacity and function of macrophages, we also examined the phenotype changes of macrophages in tumor tissue. We found an increased proportion of M1-type macrophages (CD206- ${ }^{-}$MHCII ${ }^{\text {high }} /$ total macrophage number) (figure 5I) and a decreased proportion of M2-type macrophages $\left(\mathrm{CD} 206^{+} \mathrm{MHC}\right.$ II ${ }^{\text {low }} /$ neg $/$ total macrophage number) after Sirf CAR-T cell treatment (figure 5J), indicated macrophage function might be enhanced in the treatment with Sirf CAR-T cells. These results suggested that SIRP $\alpha$-Fc produced by Sirf CAR-T cells would not only enhance the CAR-T cells persistence but also affect the TME. 
A

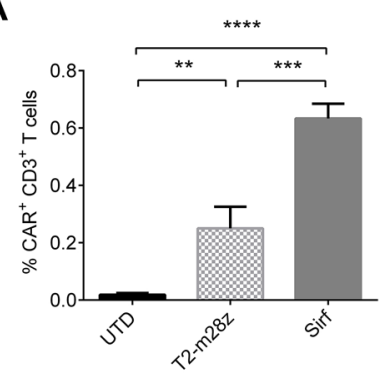

D

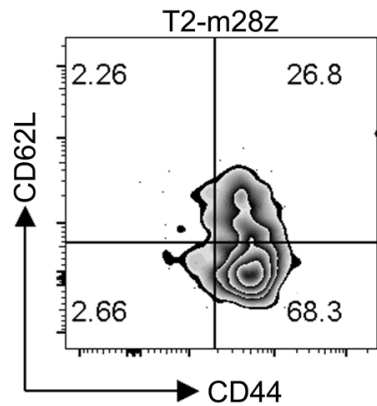

E

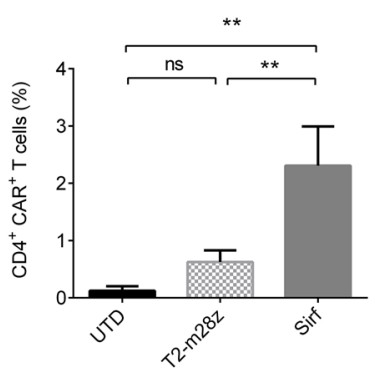

H

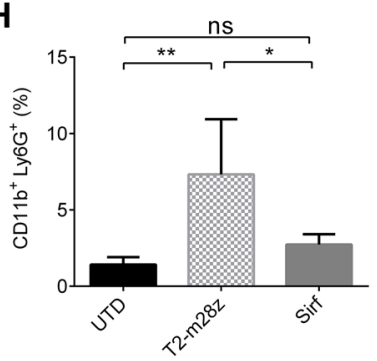

B
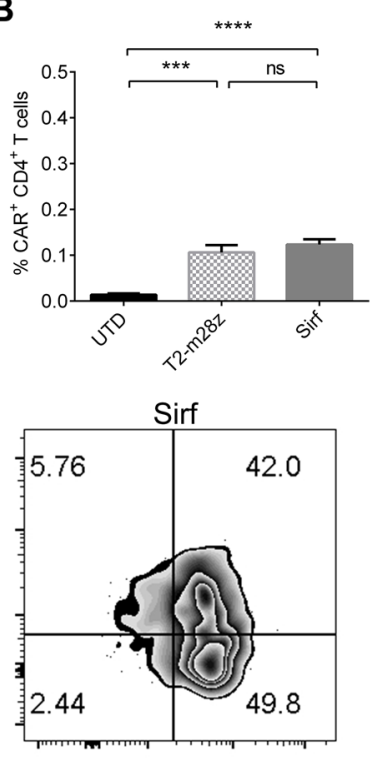

F

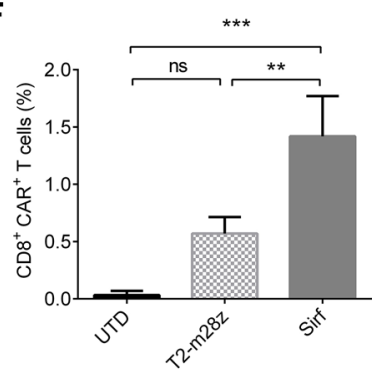

I

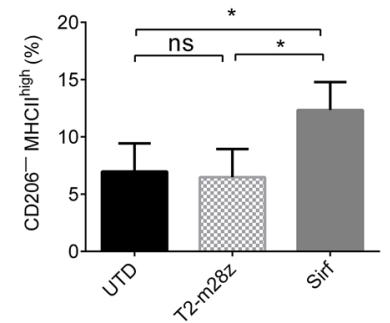

C
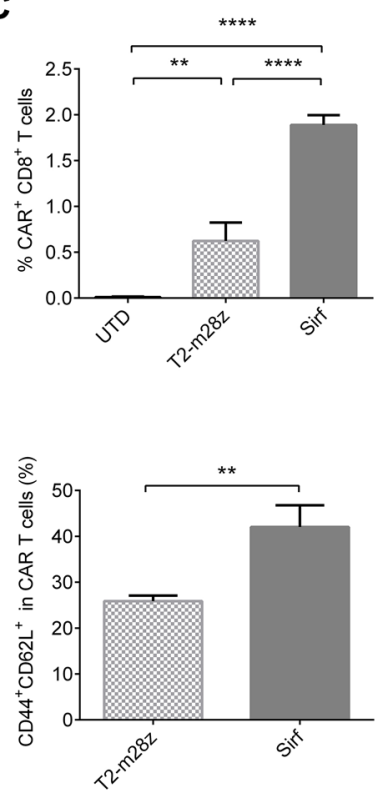

G

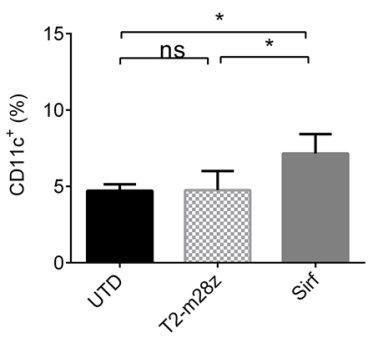

J

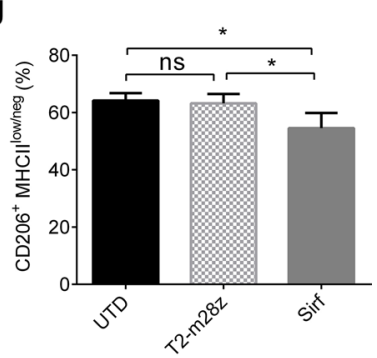

Figure 5 Secretion of SIRP $\alpha-F c$ by CAR T cells modulate the immune cells in tumor-bearing mouse. Flow cytometry was used to detect the proportion of $\mathrm{CAR}^{+}$in tumor-bearing mouse spleen. (A) CAR positive rate in $\mathrm{CD}^{+} \mathrm{T}^{\mathrm{T}}$ cells. (B) CAR positive rate in $\mathrm{CD} 4^{+} \mathrm{T}$ cells, (C) CAR positive rate in $\mathrm{CD} 8^{+} \mathrm{T}$ cells. (D) $\mathrm{CD} 44$ and $\mathrm{CD} 62 \mathrm{~L}$ of $\mathrm{CD} 3^{+} \mathrm{CAR}^{+} \mathrm{T}$ cells in mouse spleen. Flow cytometry

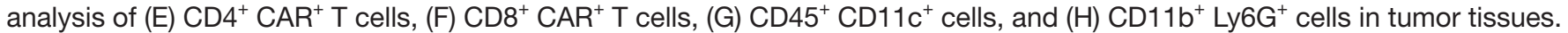
(I) Frequency of M1-like (CD206- $\left.{ }^{-} \mathrm{MHClI}^{\text {high }}\right)$ and (J) M2-like (CD206 ${ }^{+} \mathrm{MHC}$ II $^{\text {low/neg }}$ ) TAMs cell populations in $\mathrm{CD}^{2} 5^{+} \mathrm{CD}^{-}$ $\mathrm{CD} 11 \mathrm{~b}^{+} \mathrm{F} 4 / 80^{+}$in tumor tissues. All data are presented as mean $\pm \mathrm{SEM}$ from experiments, $\mathrm{N}=3$ mice/group. ${ }^{*} \mathrm{p}<0.05,{ }^{* *} \mathrm{p}<0.01$, ${ }^{* * *} \mathrm{p}<0.001,{ }^{* \star \star *} \mathrm{p}<0.0001$. CAR, chimeric antigen receptor; MHC, major histocompatibility complex; ns, not significant; SIRP $\alpha$, signal regulatory protein $\alpha$; TAM, tumor-associated macrophages; UTD, untransduced.

\section{Enhanced memory phenotype of Sirf CAR-T cells in association with decreased apoptosis}

To identify the mechanism of enhanced antitumor activity of Sirf CAR-T cells, we analyzed the changes of surface markers in different CAR-T cells after antigen stimulation with MC38-Trop2 ${ }^{+}$tumor cells. Consistent with the findings in vivo, we observed that the percentages of $\mathrm{T}_{\mathrm{CM}}$ in Sirf CAR-T cells were significantly higher than those of T2-m28z CAR-T cells (figure 6A). We next examined the apoptosis in
$\mathrm{CAR}^{+} \mathrm{T}$ cells after co-cultured with tumor cells. Less apoptotic cells were existed in Sirf CAR-T cells than in T2-m28z CAR-T cells after tumor antigen stimulation (figure 6B), but there were no differences in non-CAR-T cells which could not be stimulated by tumor antigen (figure 6C). This enhanced anti-apoptosis of CAR-T cell has also been confirmed by purified SIRP $\alpha$-Fc protein in vitro experiment (online supplemental figure S3A,B). We also found that an important immune checkpoints molecule, programmed 
A

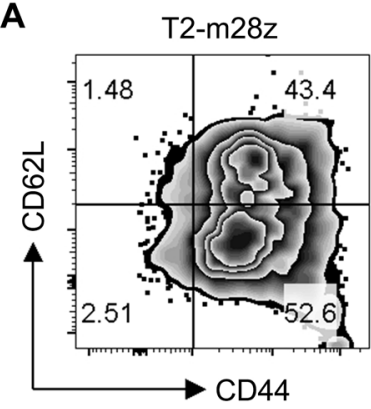

B

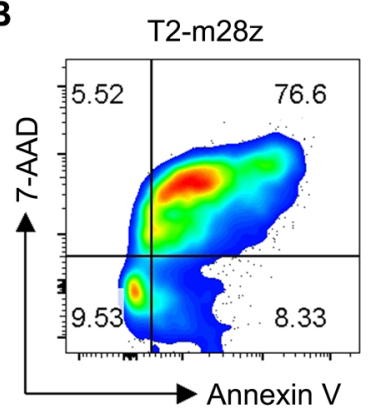

C

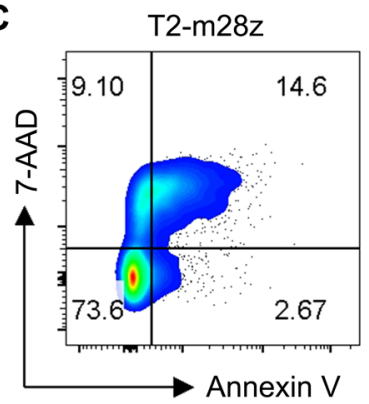

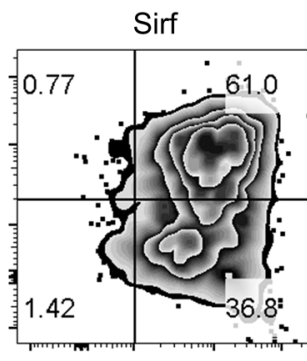
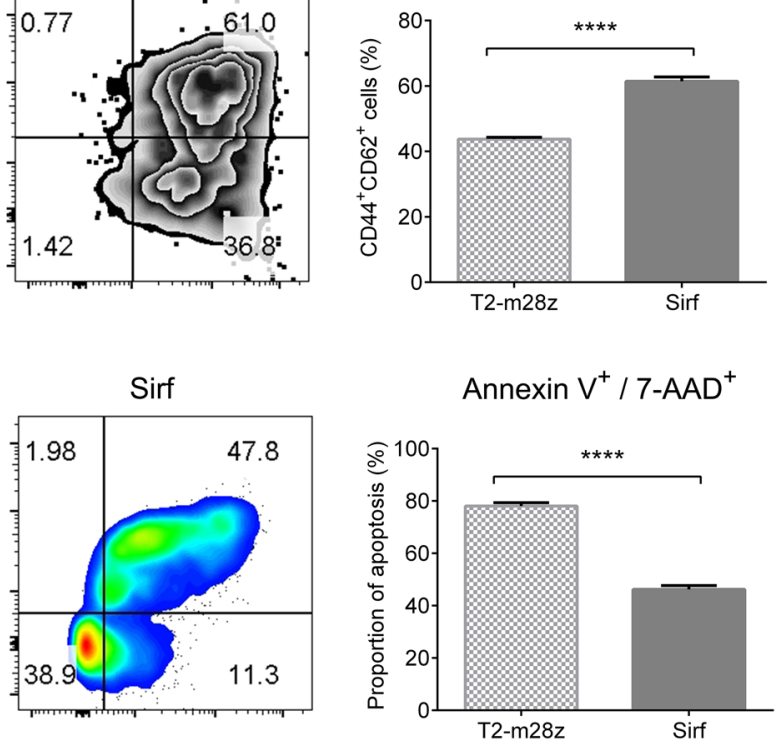

Annexin $\mathrm{V}^{+} /$7-AAD ${ }^{+}$
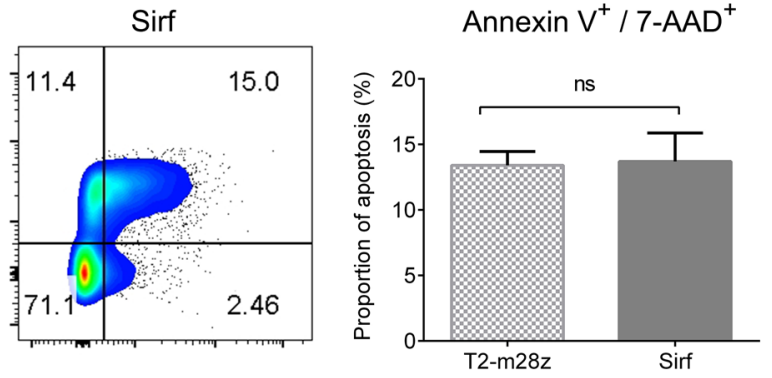

D

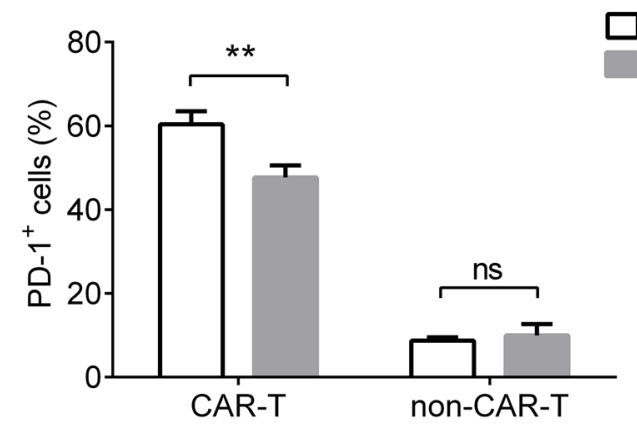

$T 2-m 28 z$

Sirf

Figure 6 The differential and apoptosis of activated CAR-T cells. The level of CAR T cells apoptosis and CD62L were analyzed by flow cytometry after co-cultured with MC38-Trop2 ${ }^{+}$for 48 hours without cytokine addition in vitro. (A) CD44 and CD62L of CAR T cells. Annexin V / 7-AAD-staining of CAR ${ }^{+} T$ cells (B) and non-CAR-T cells (C). (D) The proportion of PD- $1^{+}$cells in T cells were analyzed by flow cytometry after coculturing with MC38-Trop2 ${ }^{+}$for 48 hours without cytokine addition in vitro. All data are presented as mean \pm SEM from experiments, $\mathrm{N}=3 .{ }^{* \star} \mathrm{p}<0.01,{ }^{* * *} \mathrm{p}<0.0001$. CAR, chimeric antigen receptor; ns, not significant.

cell death 1 (PD-1), was lower expressed on Sirf CAR-T cells than on T2-m28z CAR-T cells (figure 6D). The expression of PD-1 in CAR-T cells can be effectively reduced in the presence of SIRP $\alpha$-Fc protein (online supplemental figure S3C,D), indicating that the PD-1 pathway involved in the regulation of Sirf CAR-T cells antitumor effects.

\section{DISCUSSION}

CAR-T cell therapy has shown remarkable success in hematologic malignancies but has little effect on solid tumors. The immunosuppressive microenvironment in solid tumors has been regarded as one important factor for immunotherapy, which would result in affectless of CAR-T cells in solid tumors. ${ }^{27}{ }^{28}$ In this study, we developed Sirf CAR-T cells that could produce a CD47 blocker SIRP $\alpha$-Fc fusion protein. Sirf CAR-T cells showed 
potent antitumor activity and improved therapeutic effects against solid tumors in vivo. Results showed that the secretion of SIRP $\alpha-F c$ by CAR-T cells enhanced the macrophage phagocytosis and improved the CAR-T cell persistence to apoptosis as well as reduced immunosuppression in the tumor tissues, which demonstrated the potential of Sirf CAR-T cells in solid tumor therapy.

Previous studies have demonstrated that blocking CD47 can improve the phagocytosis of antibody-modified targets by macrophages. However, the ubiquitous expression of CD47 on many cells can cause severe side effects when treated with CD47 antibodies. The expression of CD47 on red blood cells or platelets prevents attaining the requisite therapeutic dose of anti-CD47 antibodies in tumor tissue and might induce substantial anemia. ${ }^{29}$ Indeed, anti-CD47 antibodies currently focus on choosing the IgG4 molecules without Fc $\gamma$ R-binding capacity, to avoid on-target, off-tumor toxicity. ${ }^{30}$ However, most of these antibodies still induce anemia in patients. Blockade of CD47 using SIRP $\alpha$-Fc protein was also employed in clinical practice. The SIRP $\alpha$ region of SIRP $\alpha-F c$ interacts with $\mathrm{CD} 47$, while the Fc region binds to Fc $\gamma$ receptors and induces antibody-dependent cell-mediated cytotoxicity of NK cells and antibody-dependent cellular phagocytosis of macrophages. ${ }^{31}$ Compared with traditional antibodies, SIRPo-Fc fusion protein has a smaller molecular size to facilitates tissue penetration and distribution. In fact, SIRPo-Fc binds to CD47 is different from other CD47 antibodies and minimally binds to human erythrocytes. ${ }^{32}$ This unusual property could reduce the risk of anemia but still induce a high incidence of thrombocytopenia. ${ }^{33}$ The CAR-modified T cells can specifically lyse tumor cells and performance as 'living drugs' that can be used as ideal vehicles for CD47 blocker delivery through local secreting and accumulating in the TME, which could limit the severe side effects of CD47 blocker or antibodies. Based on this logic strategy, we designed Sirf CAR-T cells in this study, with which CAR T cells secreted the SIRP-Fc that could bind to CD47 and enhance the macrophage phagocytosis against tumor cells. Moreover, cytokines expressed by CAR-T cells were also beneficial for macrophage phagocytosis, which indicated that there was a synergistic effect between CD47 blockade and CAR-T cells. Our results indicated the promise of Sirf CAR-T cell therapy in solid tumors by the positive synergy of activation of CAR-T cells with CD47 blockade effects.

MDSC serves as a facilitator of tumor proliferation and immunotherapy resistance and is a major obstacle in CAR-T cell therapy. ${ }^{10}{ }^{34}$ Reduction of MDSCs could enhance the efficacy of CAR-T cells and improve immunotherapy outcomes. ${ }^{35}$ Recent studies had reported that treatment with $\mathrm{CD} 47$ antibodies in a long-term kidney allograft tolerance model induced graft dysfunction and rejection along with a significant decrease of MDSC..$^{36}$ Although these findings indicated that the SIRP $\alpha-C D 47$ axis could regulate MDSC function and contribute to immune tolerance, its mechanism remains unknown. In this study, we demonstrated that administration of Sirf
CAR-T cells decreased the MDSC cells in the tumor bed, suggesting that MDSC inhibition could be one pathway for Sirf CAR-T cells to exert their antitumor effect. Further exploration is necessary to reveal the mechanism of SIRPo-Fc fusion protein in MDSC reduction. DC is a major antigen-presenting cells and can efficiently ingest, process, and present antigens. SIRP $\alpha$ is very important for the development and maturation of DC. ${ }^{37}$ Studies have also reported that the therapeutic effects of anti-CD47 antibodies require the participation of $\mathrm{DC}$ and $\mathrm{CD}^{+} \mathrm{T}$ cells. ${ }^{23}$ In this study, there were more $\mathrm{CD} 11^{+} \mathrm{DC}$ in tumor tissues after reinfusion of Sirf CAR-T cells, indicating that DC recruitment could contribute to Sirf CAR-T cells enhancing antitumor effects.

Clinical studies indicate that $\mathrm{T}_{\mathrm{CM}}$ provides greater antitumor activity than other subsets of $\mathrm{T}$ cells to exert a longer-term antitumor effect. In contrast to the rapid proliferation of $\mathrm{T}_{\mathrm{EM}}$ cells, $\mathrm{T}_{\mathrm{CM}}$ cells have been proven to show superior persistence and antitumor activity. ${ }^{38}$ Since SIRP $\alpha$ and CD47 interaction can lead to two-way signals, which can suppress the phagocytosis of SIRP $\alpha^{+}$myeloid cells, as well as transmit signals to $\mathrm{CD} 47^{+} \mathrm{T}$ cells. CD47 is involved in the differentiation of $\mathrm{T}$ cells and may control the generation of memory T cells. ${ }^{39}{ }^{40}$ In addition, antimouse SIRP $\alpha$ antibodies could increase the frequency of $\mathrm{T}_{\mathrm{CM}}$ in lymph nodes and tumor tissue. ${ }^{41}$ However, the function of $\mathrm{CD} 47$ affecting memory $\mathrm{T}$ cells is poorly understood. In this study, the Sirf CAR-T cells treatment group displayed a higher $\mathrm{T}_{\mathrm{CM}}$ ratio in splenocytes, which demonstrated that $\mathrm{T}_{\mathrm{CM}}$ differentiation could be regulated by Sirf CAR-T cells (figure 5D). CD62L plays an important role in $\mathrm{T}$ cell homing characteristics and participates in lymphocyte recycling. ${ }^{42}$ The high expression of CD62L in $\mathrm{T}_{\mathrm{CM}}$ cells suggests that Sirf CAR-T cells have strong homing ability. We speculated that SIRP $\alpha-F c$ may regulate the migration of CAR-T cells into tumor tissue by up-regulating the expression of CD62L. Whether SIRP $\alpha-F c$ regulates the migration and homing of CAR-T cells remains to be further studied. High expression of PD-1 is regarded as the hallmark of the exhausted CD8 T cells and suppresses T cell function. ${ }^{43}$ In our study, we found attenuated PD-1 expression on Sirf CAR-T cells, neither non-CAR-T cells nor T2-m28z CAR-T cells, suggesting that SIRP-Fc protein could enhance CAR-T cell activity by downregulating inhibitory immune checkpoint molecule expression. However, the mechanism underlying SIRP-Fc protein suppresses PD-1 expression remains to be revealed in the future.

Previous reports indicated that the persistence of CAR-T cells is associated with therapeutic response. ${ }^{44}$ In this regard, the anti-apoptosis capacity of CAR-T cells may be a critical parameter for cancer treatment. SIRP $\alpha$ interactions with $\mathrm{CD} 47$ induce PKC $\theta$ translocation and synergizes with TCR ligation for $\mathrm{T}$ cell activation. Therefore, it has the potential to modulate antigen presentation to $\mathrm{T}$ cells. ${ }^{45}$ SIRP $\alpha$ is involved in DC-mediated T-cell activation and increases the sensitivity of limiting doses of antigen during T cell/APC interactions. ${ }^{46}$ TCR activation increase 
the availability of thrombospondin-1 (TSP-1) on activated $\mathrm{T}$ cells and change the conformation of the CD47 molecule, that $\mathrm{T}$ cells are more prone to apoptosis. ${ }^{45} 47$ Re-encounter with SIRP $\alpha-\mathrm{Fc}$ or SIRP $\alpha^{+}$myeloid cells might offer an advantage to avoid TSP-1-induced cell death. ${ }^{48} 49$ Studies have demonstrated that CD47 interactions with TSP-1 or SIRP-a regulate the cell death and phagocytosis of malignant cells, and CD47 antibody could induce T cell death after antigen stimulation. ${ }^{50}$ In this study, we observed that Sirf CAR-T cells improved anti-apoptosis ability on antigen stimulation (figure 6C,D). We thus propose CD47/SIRP $\alpha$ axis as an important signal to maintain homeostasis and regulate CAR-T cells apoptosis. Considering the stronger capacity of $\mathrm{T}_{C M}$ cells in antiapoptosis and persistence, more $\mathrm{T}_{\mathrm{CM}}$ populations of Sirf CAR-T cells would contribute to the potent persistence of CAR-T cells in cancer immunotherapy. More efforts need to be performed to elucidate the mechanism of SIRP $\alpha-F c$ promoting survival of Sirf CAR-T cells in future study.

In summary, Sirf CAR-T cells combined the CAR-T cell technology with the CD 47 blocker SIRP $\alpha$-Fc fusion protein, overcame the bottleneck of CAR-T cells in solid tumors therapy practice, and displayed potent antitumor effects in mouse tumor-bearing model. Sirf CAR-T cells showed antitumor efficacy improvement against solid tumors by enhancing macrophage phagocytosis, recruiting more DC into tumor tissue, increasing the proportion of $T_{C M}$ cells, and preventing the apoptosis of CAR-T cells themselves, as well as attenuating PD-1 expression on CAR-T cells. Thus, Sirf CAR-T cell therapy affected both innate and adaptive immune responses, which displayed synergized efficacy on antitumor effects against solid tumors. Our studies indicate the potential clinical application of CD47 blocked armored CAR-T cells in tumor therapy that will expand the application of CD47 and CAR-T therapy to a far broader spectrum of malignancies.

Contributors ZH and HC designed the study. HC, YY, YD, FW, QZ, BY, and ZH performed experiments, collected and analyzed data. YL and ZL provided technical support on the tumor-bearing mouse model. $\mathrm{HC}$ and $\mathrm{ZH}$ drafted the manuscript. All authors revised and approved the manuscript. $\mathrm{ZH}$ acts as the guarantor of this work.

Funding This research was supported by the Guangdong Basic and Applied Basic

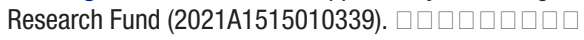

Competing interests None declared.

\section{Patient consent for publication Not applicable.}

Ethics approval The research was approved by the Zhongshan School of Medicine, Sun Yat-sen University and the Third Affiliated Hospital of Guangzhou Medical University (Guangzhou, China). This research was performed in accordance with the Declaration of Helsinki and according to national and international guidelines. All mouse experiments were approved by and followed the ethical regulations of IACUC, South China Agricultural University.

Provenance and peer review Not commissioned; externally peer reviewed.

Data availability statement No data are available. All data relevant to the study are included in the article or uploaded as online supplemental information.

Supplemental material This content has been supplied by the author(s). It has not been vetted by BMJ Publishing Group Limited (BMJ) and may not have been peer-reviewed. Any opinions or recommendations discussed are solely those of the author(s) and are not endorsed by BMJ. BMJ disclaims all liability and responsibility arising from any reliance placed on the content. Where the content includes any translated material, BMJ does not warrant the accuracy and reliability of the translations (including but not limited to local regulations, clinical guidelines, terminology, drug names and drug dosages), and is not responsible for any error and/or omissions arising from translation and adaptation or otherwise.

Open access This is an open access article distributed in accordance with the Creative Commons Attribution Non Commercial (CC BY-NC 4.0) license, which permits others to distribute, remix, adapt, build upon this work non-commercially, and license their derivative works on different terms, provided the original work is properly cited, appropriate credit is given, any changes made indicated, and the use is non-commercial. See http://creativecommons.org/licenses/by-nc/4.0/.

\section{ORCID iD}

Zhaofeng Huang http://orcid.org/0000-0003-4367-6943

\section{REFERENCES}

1 Feins S, Kong W, Williams EF, et al. An introduction to chimeric antigen receptor (CAR) T-cell immunotherapy for human cancer. Am J Hematol 2019;94:S3-9.

2 Larson RC, Maus MV. Recent advances and discoveries in the mechanisms and functions of CAR T cells. Nat Rev Cancer 2021;21:145-61.

3 Tong C, Zhang Y, Liu Y, et al. Optimized tandem CD19/CD20 CARengineered $\mathrm{T}$ cells in refractory/relapsed B-cell lymphoma. Blood 2020;136:1632-44.

4 Abramson JS, Palomba ML, Gordon LI, et al. Lisocabtagene maraleucel for patients with relapsed or refractory large B-cell lymphomas (TRANSCEND NHL 001): a multicentre seamless design study. Lancet 2020;396:839-52.

5 Khurana A, Dalland JC, Young JR, et al. Brexucabtagene autoleucel therapy induces complete remission in a primary refractory blastoid mantle cell lymphoma with neurolymphomatosis. Am J Hematol 2021;96:E298-301.

6 Neelapu SS, Locke FL, Bartlett NL, et al. Axicabtagene ciloleucel CAR T-cell therapy in refractory large B-cell lymphoma. N Engl J Med 2017;377:2531-44.

7 Roex G, Timmers M, Wouters K, et al. Safety and clinical efficacy of BCMA CAR-T-cell therapy in multiple myeloma. J Hematol Oncol 2020;13:164.

8 Marofi F, Motavalli R, Safonov VA, et al. CAR T cells in solid tumors: challenges and opportunities. Stem Cell Res Ther 2021;12:81.

9 Hirata E, Sahai E. Tumor microenvironment and differential responses to therapy. Cold Spring Harb Perspect Med 2017;7

10 Burga RA, Thorn M, Point GR, et al. Liver myeloid-derived suppressor cells expand in response to liver metastases in mice and inhibit the anti-tumor efficacy of anti-CEA CAR-T. Cancer Immunol Immunother 2015;64:817-29.

11 Ninomiya S, Narala N, Huye L, et al. Tumor indoleamine 2,3-dioxygenase (IDO) inhibits CD19-CAR T cells and is downregulated by lymphodepleting drugs. Blood 2015;125:3905-16.

12 Tang N, Cheng C, Zhang X. TGF- $\beta$ inhibition via CRISPR promotes the long-term efficacy of CAR T cells against solid tumors. JCI insight 2020;5.

13 Kasakovski D, Xu L, Li Y. T cell senescence and CAR-T cell exhaustion in hematological malignancies. J Hematol Oncol 2018;11:91.

14 Adachi K, Kano Y, Nagai T, et al. IL-7 and CCL19 expression in CAR-T cells improves immune cell infiltration and CAR-T cell survival in the tumor. Nat Biotechnol 2018;36:346-51.

15 Avanzi MP, Yeku O, Li X, et al. Engineered tumor-targeted T cells mediate enhanced anti-tumor efficacy both directly and through activation of the endogenous immune system. Cell Rep 2018;23:2130-41.

16 Liu Y, Di S, Shi B, et al. Armored inducible expression of IL-12 enhances antitumor activity of glypican-3-targeted chimeric antigen receptor-engineered T cells in hepatocellular carcinoma. J Immunol 2019;203:198-207.

17 Wang Y, Wang J, Yang X, et al. Chemokine receptor CCR2B enhanced anti-tumor function of chimeric antigen receptor $T$ cells targeting mesothelin in a non-small-cell lung carcinoma model. Front Immunol 2021;12:628906.

18 Rafiq S, Yeku OO, Jackson HJ, et al. Targeted delivery of a PD-1blocking scFv by CAR-T cells enhances anti-tumor efficacy in vivo. Nat Biotechnol 2018;36:847-56.

19 Matlung HL, Szilagyi K, Barclay NA, et al. The CD47-SIRP $\alpha$ signaling axis as an innate immune checkpoint in cancer. Immunol Rev 2017;276:145-64. 
20 Barclay AN, Van den Berg TK. The interaction between signal regulatory protein alpha (SIRP $\alpha$ ) and CD47: structure, function, and therapeutic target. Annu Rev Immunol 2014;32:25-50.

21 Barrera L, Montes-Servín E, Hernandez-Martinez J-M, et al. CD47 overexpression is associated with decreased neutrophil apoptosis/ phagocytosis and poor prognosis in non-small-cell lung cancer patients. Br J Cancer 2017; 117:385-97.

22 Iribarren K, Buque A, Mondragon L, et al. Anticancer effects of antiCD47 immunotherapy in vivo. Oncoimmunology 2019;8:1550619.

23 Liu X, Pu Y, Cron K, et al. CD47 blockade triggers T cell-mediated destruction of immunogenic tumors. Nat Med 2015;21:1209-15.

24 Postow MA, Sidlow R, Hellmann MD. Immune-related adverse events associated with immune checkpoint blockade. N Engl J Med 2018;378:158-68.

25 Weiskopf K. Cancer immunotherapy targeting the CD47/SIRP $\alpha$ axis. Eur J Cancer 2017;76:100-9.

26 Chen H, Wei F, Yin M, et al. CD27 enhances the killing effect of CAR T cells targeting trophoblast cell surface antigen 2 in the treatment of solid tumors. Cancer Immunol Immunother 2021;70:2059-71.

27 Martinez M, Moon EK. CAR T cells for solid tumors: new strategies for finding, infiltrating, and surviving in the tumor microenvironment. Front Immunol 2019;10:128.

28 Xie YJ, Dougan M, Jailkhani N, et al. Nanobody-based CAR T cells that target the tumor microenvironment inhibit the growth of solid tumors in immunocompetent mice. Proc Natl Acad Sci U S A 2019;116:7624-31.

29 Advani R, Flinn I, Popplewell L, et al. CD47 blockade by Hu5F9-G4 and rituximab in non-Hodgkin's lymphoma. N Engl J Med 2018:379:1711-21.

$30 \mathrm{Ma} \mathrm{L}$, Zhu M, Gai J, et al. Preclinical development of a novel CD47 nanobody with less toxicity and enhanced anti-cancer therapeutic potential. J Nanobiotechnology 2020;18:12.

31 Logtenberg MEW, Scheeren FA, Schumacher TN. The CD47-SIRP $\alpha$ immune checkpoint. Immunity 2020;52:742-52.

32 Petrova PS, Viller NN, Wong M, et al. TTI-621 (SIRP $\alpha$ Fc): a CD47-Blocking innate immune checkpoint inhibitor with broad antitumor activity and minimal erythrocyte binding. Clin Cancer Res 2017;23:1068-79.

33 Ansell SM, Maris MB, Lesokhin AM, et al. Phase I study of the CD47 blocker TTI-621 in patients with relapsed or refractory hematologic malignancies. Clin Cancer Res 2021;27:2190-9.

34 Kumar V, Patel S, Tcyganov E, et al. The nature of myeloid-derived suppressor cells in the tumor microenvironment. Trends Immunol 2016;37:208-20.

35 Long AH, Highfill SL, Cui Y, et al. Reduction of MDSCs with all-trans retinoic acid improves CAR therapy efficacy for sarcomas. Cancer Immunol Res 2016;4:869-80.
36 Pengam S, Durand J, Usal C, et al. SIRP $\alpha / C D 47$ axis controls the maintenance of transplant tolerance sustained by myeloid-derived suppressor cells. Am J Transplant 2019;19:3263-75.

37 Braun D, Galibert L, Nakajima T, et al. Semimature stage: a checkpoint in a dendritic cell maturation program that allows for functional reversion after signal-regulatory protein-alpha ligation and maturation signals. J Immunol 2006;177:8550-9.

38 Liu Q, Sun Z, Chen L. Memory T cells: strategies for optimizing tumor immunotherapy. Protein Cell 2020;11:549-64.

39 Rodríguez-Jiménez $\mathrm{P}$, Chicharro $\mathrm{P}$, Llamas-Velasco $\mathrm{M}$, et al. Thrombospondin-1/CD47 interaction regulates Th17 and Treg differentiation in psoriasis. Front Immunol 2019;10:1268.

40 Van VQ, Raymond M, Baba N, et al. CD47(high) expression on CD4 effectors identifies functional long-lived memory T cell progenitors. $J$ Immunol 2012;188:4249-55.

41 Gauttier V, Pengam S, Durand J, et al. Selective SIRP $\alpha$ blockade reverses tumor $\mathrm{T}$ cell exclusion and overcomes cancer immunotherapy resistance. J Clin Invest 2020;130:6109-23.

42 Gattinoni L, Lugli E, Ji Y, et al. A human memory T cell subset with stem cell-like properties. Nat Med 2011;17:1290-7.

43 Pauken KE, Wherry EJ. Overcoming T cell exhaustion in infection and cancer. Trends Immunol 2015;36:265-76.

44 Guedan S, Posey AD, Shaw C, et al. Enhancing CAR T cell persistence through ICOS and 4-1BB costimulation. JCl Insight 2018;3.

45 Rebres RA, Green JM, Reinhold MI, et al. Membrane raft association of CD47 is necessary for actin polymerization and protein kinase $C$ theta translocation in its synergistic activation of T cells. J Biol Chem 2001:276:7672-80.

46 Seiffert M, Brossart P, Cant C, et al. Signal-regulatory protein alpha (SIRPalpha) but not SIRPbeta is involved in T-cell activation, binds to CD47 with high affinity, and is expressed on immature CD34(+) CD38(-) hematopoietic cells. Blood 2001;97:2741-9.

47 Li SS, Liu Z, Uzunel M, et al. Endogenous thrombospondin-1 is a cell-surface ligand for regulation of integrin-dependent T-lymphocyte adhesion. Blood 2006;108:3112-20.

48 Sarfati M, Fortin G, Raymond M, et al. CD47 in the immune response: role of thrombospondin and SIRP-alpha reverse signaling. Curr Drug Targets 2008;9:842-50.

49 Van VQ, Baba N, Rubio M, et al. CD47(low) status on CD4 effectors is necessary for the contraction/resolution of the immune response in humans and mice. PLoS One 2012;7:e41972.

50 Manna PP, Frazier WA. The mechanism of CD47-dependent killing of T cells: heterotrimeric Gi-dependent inhibition of protein kinase A. $J$ Immunol 2003;170:3544-53. 